DOI: $10.25100 /$ pfilosofica.v0i53.11526

\title{
LA NOCIÓN DE CUERPO EN JUDITH BUTLER Y ROSI BRAIDOTTI
}

Iván Eduardo Díaz Peña

\section{Resumen}

Este trabajo busca presentar el debate alrededor de la materialidad del cuerpo en dos grandes teorías feministas, a saber, las teorías feministas de la diferencia sexual y la teoría feminista alrededor del género. En ese sentido, mi interés es mostrar cómo, desde una distinta aproximación al concepto de cuerpo, se articulan unas diferencias para reivindicar las luchas feministas. Quisiera en particular acercarme a las pensadoras Judith Butler y Rosi Braidotti, que tienen una articulación distinta del sujeto-cuerpo del feminismo y el lugar que este debe ocupar en las transformaciones sociales $y$ políticas. Este trabajo no pretende inclinarse por mostrar las ventajas de una teoría sobre la otra sino más bien establecer sus diferencias.

Palabras clave: feminismo; cuerpo; materialidad; posestructuralismo; género.

Cómo citar este artículo: Díaz Peña, I. E. (2021). La noción de cuerpo en Judith Butler y Rosi Braidotti. Praxis Filosófica, (53), 225-238. https://doi.org/10.25100/pfilosofica.v0i53.11526

Recibido: 25 de abril de 2021. Aprobado: 20 de junio de 2021. 


\title{
The notion of the body in Judith Butler and Rosi Braidotti
}

\author{
Iván Eduardo Díaz Peña ${ }^{1}$
}

\begin{abstract}
This work seeks to present the debate around the materiality of the body in two great feminist theories, namely, feminist theories of sexual difference and feminist theory around gender. In this sense, my interest is to show how, from a different approach to the concept of the body, they articulate differences to vindicate feminist struggles. I would particularly like to get closer to the thinkers Judith Butler and Rosi Braidotti, who have a different articulation of the subject-body of feminism and the place it should occupy in social and political transformations. This work does not pretend to be inclined to show the advantages of one theory over the other, but rather to establish their differences.
\end{abstract}

Palabras clave: Feminism; Body; Materiality; Postructuralism; Gender.

${ }^{1}$ Filósofo y abogado de la Universidad de los Andes. Magister en filosofía de la Universidad de los Andes. Docente de la facultad de ciencias jurídicas y políticas de la Universidad el Bosque. Abogado de la Comisión Colombiana de Juristas. Sus áreas de investigación son derechos humanos, feminismo, violencia y paz.

ORCID: 0000-0002-1852-1862 E-mail: iediazp@unbosque.edu.co 


\title{
LA NOCIÓN DE CUERPO EN JUDITH BUTLER Y ROSI BRAIDOTTI
}

\author{
Iván Eduardo Díaz Peña \\ Universidad El Bosque, Bogotá, Colombia
}

\section{Introducción}

A mediados del siglo XX el feminismo de segunda ola ${ }^{2}$ tomó fuerza y empezó su lucha por el igual reconocimiento de la mujer en un mundo masculino. Cixous y Beauvoir son fieles representantes de esta corriente feminista que afirma que la forma representación del mundo no solo no es neutral, sino que ha estado dominada por una lógica masculina. El hombre es el que le ha dado una cierta estructura al mundo, el hombre lo ha constituido. En ese sentido, incluso la creación del lenguaje es producto de esta forma de representación del mundo. Frente a esta forma de representación, estas filósofas alzaron su voz pues se dieron cuenta de lo problemático que era, una única representación masculina. Para Cixous, por ejemplo, las representaciones del mundo han sido únicamente binarias, dejando a la mujer con del lado del binario menos favorable (Cixous, 1995). Su lucha era frente a una representación masculina que construyó sociedades para el triunfo del macho.

La conocida frase de Beauvoir, "no se nace mujer, sino que se llega a serlo" permitió que el feminismo tomara fuerza y comenzara a comprender que el cuerpo de la mujer es construido históricamente. Uno de los debates centrales en esta época del feminismo fue si en realidad hay algo construido o algo esencial al cuerpo la mujer. En este debate, se comenzó a hablar de

\footnotetext{
${ }^{2}$ Por feminismo de segunda ola entiendo la corriente feminista que se desarrolló después del movimiento sufragista.
} 
"género" como una categoría que permitía establecer diferencias entre lo natural y lo construido. En efecto, el género se comprendió como aquella categoría que respondía a una construcción, tanto personal como social, mientras que el sexo era esa categoría que respondía a lo natural, a lo biológico. Se afirmaba que el sexo, dado que era biológico y natural era inmodificable y estático. El género por su parte, no era estático y no dependía de algo natural y biológico. En ese sentido, el género se convirtió en la categoría que rompía el nexo causal entre sexo y genero (hembra- mujer). Así las cosas, el género femenino se convirtió en la categoría universal que movilizaba la lucha feminista.

La crisis de este feminismo de segunda aparece cuando se trata de responder el "qué" es un sujeto determinado y indudablemente se cae en una lógica esencialista. El "qué" supone que hay algo determinado y natural en un sujeto. Cuando se trataba de responder qué era aquello que identificaba a la mujer como mujer, se acudía a categorías, a cualidades: la mujer es amor, la mujer es cariño, la mujer es fuerza. El "qué" cae en el defecto de clasificar por elementos que constituyen un determinado sujeto, ubica y siempre delimita. Estas problemáticas dieron lugar a nuevas corrientes dentro del feminismo como teorías queer, teorías poscoloniales y estudios postestructuralistas.

Entre las pensadoras más influyentes se encuentran Judith Butler, Haraway, Teresa de Lauretis, Beatriz Preciado, entre otras. Estas autoras se apartan de una visión esencialista o pre-lingüística del género hacia una noción transversal de las diferencias. A esta nuevas corrientes les interesa el entrecruzamiento transversal de los sujetos y los cuerpos. Esto es, que el sujeto debe ser repensado y transformado desde una nueva aproximación a la corporalidad. Es por ello que aparecen otros términos que reivindican unas categorías no esencialistas. El sujeto debe comprenderse por una multiplicidad de identidades, no binarias, que ponen en jaque la categoría de hombre y mujer. La aproximación a estas corrientes de pensamiento de clara herencia postestructuralista permite introducir nuevas preguntas sobre la identidad de los sujetos.

Así las cosas, el presente trabajo pretende abordar el concepto de cuerpo y su relación con las practicas políticas que pueden desestabilizar las categorías esencialistas y predeterminadas que moldean un sujeto. En la primera parte de este trabajo, me acercaré a la postura de Judith Butler para analizar su concepto de materialidad del cuerpo y el rasgo performativo del lenguaje. En el segundo lugar, presentaré la postura de Rosi Braidotti que se acerca a la materialidad del cuerpo desde su concepto de la diferencia 
sexual y lo articula con una experiencia de la corporización de la experiencia humana.

\section{El cuerpo en el posestructuralismo feminista}

Una de las premisas centrales del debate entre esencialismo y constructivismo es que hay algo natural y algo construido en los cuerpos. En este debate se plantean principalmente dos preguntas. La primera consiste en trazar el límite entre lo esencial y lo construido, es decir, qué era esencial y qué era construido en los cuerpos. La segunda, consiste en indagar si existe una relación de causalidad entre aquello esencial y aquello construido. En términos concretos consiste en preguntar si un cuerpo al que se le identifica como macho se debe identificar como hombre.

El movimiento postestructuralista hace una comprensión de todas las identidades excluidas de los parámetros de normalidad y pretende sacarlas de esa exclusión. En opinión de Beatriz Preciado es un movimiento postidentitario pues no defiende una identidad más, sino que se aparta críticamente de cualquier pretensión identitaria, que cae siempre en un círculo de exclusión. Estos movimientos retoman la fuerza de la exclusión y se presentan como un movimiento político, en palabras de Preciado:

Pretendía reunir todas las señas de lo abyecto. Pero la palabra servía en realidad para trazar un límite al horizonte democrático: aquel que llamaba a otro "queer" se situaba a sí mismo sentado confortablemente en un sofá imaginario de la esfera pública en tranquilo intercambio comunicativo con sus iguales heterosexuales mientras expulsaba al "queer" más allá de los confines de lo humano. Desplazado por la injuria fuera del espacio social, el "queer" estaba condenado al secreto y a la vergüenza (Preciado, 2012).

Para desmontar el esencialismo identitario, el posestructuralismo analiza y pone en tela de juicio la ontología de las identidades. Judith Butler critica la lógica identitaria pues, desde su punto de vista, ha reafirmado el imperativo heterosexual. Una de las posturas centrales de Butler es que el cuerpo es un marco de relaciones dinámicas que interactúa en un espacio ya político y social. Tanto el género y el sexo son performativos puesto que se construyen por medio de los efectos del discurso. En el libro de Butler titulado "género en disputa", ella expone como el género ha sido una construcción del sistema heterosexual. En ese sentido, entiende que el género no es una simple construcción social, sino que es una construcción social hegemónica que mantiene y reproduce los binarios. 
Los planteamientos de Judith Butler nos permiten comprender que no existe una distinción entre género y sexo. Es decir, que no hay algo así como el sexo biológico y el género como lo construido. Para poder superar esta distinción, Butler introduce su noción de cuerpos socialmente construidos. Para Butler pensar en una distinción entre sexo y género nos lleva a suponer que hay algo dado, que hay algo inmodificable, algo que no está permeado culturalmente, dice Butler:

\begin{abstract}
¿Acaso los hechos aparentemente naturales del sexo tienen Lugar discursivamente mediante diferentes discursos científicos supeditados a otros intereses políticos y sociales? Si se refuta el carácter invariable del sexo, quizás esta construcción denominada «sexo» este tan culturalmente construida como el género; de hecho, quizá siempre fue género, con el resultado de que la distinción entre sexo y género no existe como tal (Butler, 1999, p. 54).
\end{abstract}

La teoría Butler rechaza toda pregunta esencialista de los cuerpos. En este nuevo movimiento no se trata de responder cuál es la esencia del hombre o de la mujer (que hace que este cuerpo sea hombre o sea mujer), sino de comprender que es una construcción social por medio de la diferencia. Tampoco se trata de decir que todo es socialmente construido, pues esta afirmación traería consigo una sería de interrogantes: ¿Quién construye ese sujeto? ¿No hay un sujeto que construye? ¿Hay un lugar prelinguistico del sexo? Le teoría de Butler no piensa que todo es una construcción social, sino que nosotros, nuestros cuerpos y nuestros actos son parte de una cultura. En otras palabras, no se puede pensar en los cuerpos sin cultura, solo hay movimientos culturales de cuerpos: no hay cuerpos que no sean socialmente construidos.

En su libro "Cuerpos que importan" Butler pretende trabajar esta cuestión y para ello aborda la relación entre materialidad y discurso. Una de las preguntas de las que surge la discusión es ¿Qué es lo material o lo real del cuerpo? y ¿Qué es lo construido, lo socialmente producido del cuerpo? Generalmente tendemos a pensar que el cuerpo es algo natural, algo biológico. Frente a esto Butler quiere argumentar que los cuerpos también son socialmente construidos, productos de unas dinámicas de poder. Esto significa que la materialidad del cuerpo es histórica, es decir, depende de ciertos discursos hegemónicos. 
Por ello, Butler sostiene que es necesario hacer una deconstrucción ${ }^{3}$ del término materialidad. Al cuestionar el término materialidad no pretende eliminarlo del debate político, sino más bien constituir una nueva genealogía por la que pueda transitar. Para ello, debe retornarse a la historia, a la historia de la materialidad. Deconstruir la materia no implica que el término pierda su utilidad. Al hacer una deconstrucción del término se descubre que la materia está rodeada por los discursos de poder. En efecto, el concepto materia ya está constituido por una serie de violaciones, en palabras de Butler, lo que hace imposible encontrar una materia anterior al discurso. Precisamente lo que hace Butler es probar que una materialidad "anterior" o pre discursiva no existe, sino que es fruto de una visión de mundo.

La tesis que quiere sostener Butler "es que las normas reguladoras del sexo obran de una manera performativa para constituir la materialidad de los cuerpos y, más específicamente, para materializar el sexo del cuerpo, para materializar la diferencia sexual en aras de consolidar el imperativo heterosexual" (Butler, 2002, p. 18). Para entender la tesis, es necesario acudir a una primera definición de performatividad: "una práctica reiterativa y referencial mediante la cual el discurso produce los efectos que nombra" (Butler, 2002, p. 18). Esto significa que las normas discursivas operan de manera performativa, materializando el cuerpo y su sexo.

Al analizar esta cuestión, se ha dicho que lo social imprime la huella a lo natural y que por ello lo natural adquiere valor en la medida que ha sido permeado por lo social. El efecto de esta argumentación es que lo social anula lo natural: "lo que ocurre es, no solo que el sexo es absorbido por el género, sino que el sexo llega a ser algo semejante a una ficción, tal vez una fantasía, retroactivamente instalada en sitio prelingüístico al cual no hay acceso directo" (Butler, 2002, p. 23). Butler quiere argumentar que el sexo no es natural, la consideración del sexo como algo natural y anterior al discurso es efecto del poder performativo del género.

Para Butler, el debate se puede traducir en una discusión entre el esencialismo y el constructivismo. Sin embargo, Butler no quiere situarse en ningún lado de esta discusión. Para ella, este debate generó muchos problemas, ¿Quién construye ese sujeto? ¿No hay un sujeto que construye? ¿Hay un lugar prelingüístico del sexo? Muchas de las críticas que se le hicieron Butler por su libro "El género en disputa" fue que precisamente se hizo de un lado del debate, asumiendo una posición totalmente constructivista. Los críticos le señalaron a Butler que no tuvo en cuenta la

\footnotetext{
${ }^{3}$ La deconstrucción para Butler no es hacer una crítica que destruye el concepto sino hacer una crítica de un concepto necesario. Deconstruir un concepto, en consecuencia, es hacer una crítica de un concepto útil, "sin el cual no podemos hacer nada".
} 
materialidad del cuerpo, el cuerpo corporal que ocupa un espacio. Frente a ello, Butler escribe este libro para rescatar la materialidad del cuerpo, pero no una simple materialidad dada, sino una materialidad producto de los discursos y el poder.

Otro problema que el debate entre esencialismo y constructivismo pasan por alto es la cuestión de la deconstrucción. Para esta filósofa, la deconstrucción tiene que ver con la fuerza constitutiva de la exclusión. Lo abyecto tiene una fuerza constitutiva en términos de legitimidad discursiva que el debate entre constructivismo y esencialismo pasan por alto. En la construcción del género y en la articulación de su discurso, lo humano se define a partir de lo no humano. La construcción de género apela a lo excluido, a lo contrario, que el construccionismo no puede dar cuenta. En consecuencia, Butler apela a la deconstrucción como forma de apelar a la fuerza constitutiva de la exclusión, como posibilidad de eliminar las barreras del discurso legítimo. La deconstrucción es ese proceso de juego diferencial en que lo abyecto va penetrando el discurso hegemónico. Lo excluido muestra que las categorías del discurso hegemónico no aplican como se pensaba y he ahí el poder de lo excluido de replantear esas categorías.

Siguiendo los planteamientos de Irigaray, Butler hace una deconstrucción de las oposiciones binarias que han llegado a funcionar en virtud de una exclusión de un campo de posibilidades. Esas oposiciones binarias funcionan en virtud de un falogocéntrismo que ha excluido a lo femenino. Irigaray, sostiene que lo femenino ha sido lo excluido en esa oposición pues lo femenino es aquello que no puede ser representado en términos filosóficos. En consecuencia, lo que le interesa a Irigaray es retornar a ese lenguaje del cual fue excluido, apropiarse de lo que le es impropio. La exclusión de lo femenino se da precisamente en la formulación del concepto de materia. En el binario materia/forma se constituye una materialidad adicional que no corresponde a la materia: es un deposito, una articulación sobre la cual descansa el binario que Platón llama receptáculo (una chora).

Lo clave es comprender que en esa articulación sobre la que descansa la metafísica occidental, la chora es un lugar excluido que se asigna a lo femenino. En otras palabras, lo femenino no tiene lugar en la oposición binaria, sino que opera como la base del discurso, pues lo masculino abarca a los dos conceptos(materia/forma). Por lo tanto, lo femenino queda reducido a lo ininteligible, no puede decirse que es algo, no puede clasificarse o definirse dentro de la estructura falogocentrica. El receptáculo aparece en Platón en el Timeo como el lugar donde la materia adopta su respectiva forma, pero no se apropia de ella, es decir aquel lugar sin forma ni materia. 
El discurso falogocéntrico produce ese lugar en el que se constituye una materia excesiva, esto es, que está más allá de la distinción materia/forma.

Ahora bien, lo que quiere hacer Butler es una deconstrucción de este sistema filosófico en el que muestre que eso que fue excluido en realidad opera como un exterior necesario, lo excluido opera con posibilidades de constitución. En otras palabras, le femenino sería un exterior que permite el discurso falogocéntrico, no es un exterior absoluto sino un exterior que constituye. Así, ella propone, al igual que Irigaray, que la filosofía debe hacer un trabajo "volver a atravesar" de forma retórica el falogocéntrismo filosófico. Un volver a atravesar que Irigaray lo establece en termino de mimesis. Si las mujeres saben que están imitando es porque saben que participan de algo imitado, algo ficticio. Para Irigaray, el exceso en la imitación es lo que puede quebrar esa repetición del sistema falogocéntrico.

\section{Una aproximación al concepto de cuerpo desde la diferencia sexual}

Quisiera ahora traer a colación una pensadora que desde el concepto de la diferencia sexual ha tratado de pensar lo femenino como herramienta transformadora del patriarcado. Rosi Briadotti hace una aproximación desde el posestructuralismo y lo entrecruza con el asunto de la diferencia sexual. El concepto de la diferencia sexual surge de los análisis de Luce Irigaray en donde revela la identidad entre el logos y la masculinidad, en el cual la mujer es lo nombrado, es el objeto del discurso. Por medio del lenguaje se significó y excluyó a la mujer del plano de lo nombrarle: es lo otro. En ese sentido, la mujer debe hacer una deconstrucción de las configuraciones que le son impuestas y nombrarse a sí misma. Braidtotti retoma estas ideas y las conjuga con las experiencias de la posmodernidad para señalar que el sujeto está constituido por un haz de contradicciones y multiplicidades. (Palaisi, 2018, p. 64)

Esto significa que la experiencia de la mujer, marcada por la diferencia sexual, debe ser el origen de las transformaciones políticas. En ese sentido, lo primero que hay que decir, es que los cuerpos femeninos siempre han sido caracterizados como "lo otro", compuestos a su vez por variables culturales, sociales, económicas y políticas. Esas características que comparten deben ser el punto de partida desde el cual se piensen las experiencias de vida de las mujeres. Por eso dice Braidotti: "Pensar a través del cuerpo en lugar de la huida del mismo" (Braidotti, 2005, p.18). Desde el comienzo de su texto "Metamorfosis", Braidotti toma distancia de Butler afirmando "Butler opta por el giro lingüístico, yo tomo la senda nómada de la carne" (Braidotti, 2005, p. 67). Para Braidotti debe haber una apropiación de los afectos y el 
deseo de las mujeres. Este rasgo afectivo toma una relación fundamental frente al pensamiento de la diferencia sexual.

Para esta autora toda la filosofía siempre ha estado ligada a una metafísica de la presencia. Es decir, que el mundo filosófico ha establecido una constante dicotomía en la que la verdad, lo inteligible, el logos pertenece a un campo o categoría inaccesible para los seres humanos. En cambio, lo femenino está asociado a lo natural, lo material, lo sensible y accesible (Ávila, 2014, p. 171). Por ello, fiel a su herencia posestructuralista, propone una nueva aproximación conceptual, en la que las mujeres modifican y reafirman las fuerzas existentes, modulan sus identidades y reafirman nuevas posibilidades de existencia. Retoma la tesis de critica al sujeto racional y para ello propone el concepto de sujeto nómade.

El sujeto, para Braidotti, está formado por deseos inconscientes y estos se conjugan con la figura del devenir. El proceso de devenir mujer reconfigura la subjetividad de lo que es y se propone como un sujeto que siempre puede llegar a ser. El proceso de devenir mujer, aunque opera de forma inconsciente, no es una diferencia cualquiera, sino que es una diferencia estructural en el desarrollo de un cuerpo. Braidotti señala en su libro "Metamorfosis" que puede identificar dos posturas dentro del feminismo contemporáneo: El feminismo postestructuralista de la diferencia sexual y el feminismo postestructuralista anglosajón. Para el materialismo de la diferencia sexual, "la materialidad está encarnada donde la sexualidad es entendida como un proceso y un elemento constitutivo" (Hernández, 2007, $\mathrm{p}, 84)$. La experiencia de la diferencia sexual debe ser analizada desde su aspecto positivo y no negativo. Dice Braidotti:

"el cuerpo viene a ser una interacción compleja de fuerzas sociales y simbólicas sofisticadamente construidas: no es una esencia, y mucho menos una sustancia biológica, sino un juego de fuerzas, una superficie de intensidades, puros simulacros sin originales" (Braidotti, 2005, p. 37).

La intención de Braidotti es explorar como el ser humano no es una simple materialidad corporal, sino que está atravesado por una experiencia afectiva. La noción de cuerpo que propone Braidotti está mediada por la idea de la diferencia sexual, porque para ella, este concepto permite identificar, analizar y codificar las experiencias diversas que atraviesan los cuerpos. Para Braidotti: "la diferencia sexual esta inscrita en el cuerpo de mil maneras distintas" (Braidotti, 2005, p. 67). Es decir que la materialidad tiene que ver con un cuerpo encarnado. De allí que, extraen que la multiplicidad es reconocer un cierto carácter positivo de la diferencia sexual por lo diverso 
de las figuraciones que atraviesan la experiencia humana. La potencia de la diferencia sexual radica en que reconoce las contradicciones y multiplicidades que operan dentro de un mismo sujeto.

El sujeto del feminismo es un sujeto que ya está compuesto por su género y sexualidad, está encarnado a través de la diferencia sexual. Es decir que Braidotti intenta pensar cómo un cuerpo materializa sus experiencia humana a través de los rasgos que lo constituyen. Estos cuerpos no solo han sido moldeados ya por rasgos de la diferencia sexual sino que interactúan espacio temporalmente con otras variables de su entorno. En ese sentido, el concepto de la diferencia sexual no es el único componente que determina un cuerpo femenino, pero sí le permite a las mujeres tener una experiencia común.

Braidotti hace una nueva aproximación al concepto de cuerpo también desde su articulación del sujeto nomade. El sujeto, que ya es un sujeto deseante, afectivo e imaginativo se conjugan para construir una subjetividad política: el nomadismo (Palaisi, 2018, p. 65). Este sujeto se caracteriza por su capacidad subversiva y desestabilizadora de categorías absolutas o que permanecen un largo espacio de tiempo:

El cuerpo, o la corporización del sujeto, no debe entenderse ni como una categoría biológica ni como una categoría sociológica, sino más bien como un punto de superposición entre lo físico, lo simbólico y lo sociológico. En otras palabras, el énfasis feminista en la corporización va de la mano con el repudio radical del esencialismo (Braidotti, 2000, p. 30).

El sujeto nomade deja atrás cualquier figuración, deseo o afecto hacia lo ya establecido y predeterminado. En el proceso de construcción de la identidad humana, esta no está definida previamente, sino que se moldea por las múltiples interacciones en que una experiencia se desarrolla. A pesar de que la identidad de un sujeto esté siempre en transito, se puede identificar una localización y sus posibilidades transformadoras. No significa entonces que la experiencia de ser mujer se determina biológicamente, sino que es en el orden simbólico del mundo patriarcal que se ha determinado las rasgos que deben seguir. Por ello, el feminismo debe hacer una reapropiación del concepto y movilizarlo como eje de reivindicación de las apuestas de las mujeres.

Braidotti comprende que la materialidad del cuerpo radica en su realidad múltiple, su dinamismo, su fluidez, su capacidad multisensorial. Por ende, reafirma una nueva materialidad que puede subvertir las bases de la metafísica occidental. Esto porque reafirma que el cuerpo sea una mera construcción natural o biológica, sino que el cuerpo es un constante 
devenir: "el cuerpo viene a ser una interacción compleja de fuerzas sociales y simbólicas sofisticadamente construidas: no es una esencia, y mucho menos una sustancia biológica, sino un juego de fuerzas, una superficie de intensidades, puros simulacros sin originales" (Braidotti, 2005, p. 37). En suma, el cuerpo un complejo fruto de la interacción de fuerzas que lo moldean continuamente.

Uno de los aspectos que le interesa señalar a esta autora es que el cuerpo no es un mero receptor de la cultura y la sociedad, como podría ser para Butler, sino que el cuerpo tiene una materialidad que no es totalmente biológica. El cuerpo es un espacio de conexión con otros sujetos, con otros entornos y con su propia naturaleza:

Elcuerpo continúa siendoun haz de contradicciones: es una entidadbiológica, un banco de datos genéticos y, a la vez, también continúa siendo una entidad biosocial, es decir, un fragmento de memorias codificadas, personalizadas. En este sentido, es parte animal y parte máquina, pero la oposición dualista entre ambas, que nuestra cultura ha asumido desde el siglo XVIII como modelo dominante, es hoy inadecuada (Braidotti, 2005, p. 37).

El cuerpo que ya ha sido corporizado por medio de pensamientos es el sujeto nómade del feminismo. La multiplicidad de factores que afectan un cuerpo no pueden representar por sí mismo lo femenino. Es por ello que, como lo femenino es lo no representable, es también aquello que tiene la posibilidad de manifestarse y hacer reivindicaciones políticas y desconocer las múltiples configuraciones que identidad al sujeto como una mismidad:

Si "esencia" significa la sedimentación histórica de productos discursivos multiestratificados, ese cúmulo de definiciones, exigencias y expectativas culturalmente codificadas sobre las mujeres, o sobre la identidad femenina -ese repertorio de ficciones reguladoras tatuadas en nuestras pieles-, entonces sería falso negar no sólo que esta esencia exista sino, también que es poderosamente operativa (Braidotti, 2005, p. 61).

En ese sentido, para esta pensadora lo femenino tiene una potencia transformadora por su diferencia sexual. Es allí donde encuentra una posibilidad de manifestarse y hacer reivindicaciones políticas. Su fuerza está en desujetarse de todas esas categorías que modulan y configuran cualquier sujeto. Es por esta razón que el concepto de diferencia sexual tiene efectos simbólicos en los cambios políticos. Todo cuerpo sexuado es un punto de 
partida desde el cual se pueden construir nuevas subjetividades que siempre estén en proceso de transformación.

La transformación del sujeto siempre debe ocurrir a través del cuerpo y esta siempre debe ser dinámica a través de los múltiples relacionamientos con otros cuerpos. No hay una identidad natural al cuerpo sino que depende de la interacción y movimientos que lo componen (Hernández, 2017, p. 24). El cuerpo, a pesar de que siempre está en una horizonte de localización y en un juego de fuerzas que lo componen, puede desplegar una potencia afectiva que reconfigure todas las coordenadas de sentido.

En suma, Braidotti propone una nueva aproximación a que sea lo femenino lo que pueda reapropiarse y resignificarse para combatir el patriarcado. En esta reconfiguración, el cuerpo surge como una herramienta política que trasciende la noción del sujeto racional masculino. Partir de esta materialidad de la diferencia sexual entre los cuerpos de hombre y mujeres rompe con la estructura del esencialismo y le permite hacer una reaproximación desde el cuerpo a nuevas relaciones colectivas y comunitarias entre las mujeres y sus cuerpos.

\section{Conclusión}

En cualquier caso, las posiciones presentadas nos permiten pensar un nuevo relacionamiento con el mundo no solo a través de la razón, sino de la experiencia corporal. Articulan una nueva capacidad reconfigurativa de los cuerpos porque, aunque están atravesados por esas estructuras de dominación, pueden modular y reconfigurar esos quiebres. Cualquier cuerpo, en ese sentido, puede reclamar la posibilidad de actuar políticamente. Los cuerpos dominados, sujetados, excluidos, pueden confrontar la dominación y la sujeción.

El cuerpo, en estas nuevas comprensiones feministas, no es un cuerpo totalmente sujetado o pasivo, en los términos de Braidotti, sino que es un cuerpo que puede resignificarse. El sujeto de la acción, en efecto, son los cuerpos que pueden reconfigurar las condiciones políticas. La capacidad reside no en el sujeto racional, unitario y masculino, sino que es el cuerpo el que puede atravesar las asignaciones que lo modulan. Con ello, el énfasis de Butler en que en la manifestación política se da por medio de la corporalidad y con ello, los cuerpos están arrojados en una ontología relacional: soy un cuerpo que comparto espacio con otros cuerpos

En últimas, estas autoras se debaten por el sujeto capaz de transformaciones políticas y la necesidad de pensar esta transformación a través del cuerpo. Las marcadas diferencias entre ambas autoras, más que estancar los propósitos de la lucha feminista, posibilitan nuevas miradas 
y aproximaciones que siempre son útiles en cualquier movimiento del pensamiento. Pienso que Butler sigue comprendiendo que el concepto de la diferencia sexual, por más que se intente desligarlo, es fruto de un eje patriarcal. Es decir, que para Butler pensar la diferencia sexual ya conlleva una especie de materialismo esencialista del que no podemos escapar. Esto significa que la pregunta por la diferencia sexual necesariamente atraviesa la pregunta por lo biológico y lo construido de un cuerpo, que conlleva a su vez a un círculo sin salida.

Por su parte, para Braidotti la diferencia sexual debe ser eje de transformación del feminismo por medio de una reapropiación de los cuerpos afectivos. Es el lugar desde el cual las mujeres pueden constituir nuevas relaciones con ellas mismas y dentro de la sociedad. Se convierte en un eje transformador, que, alejado de cualquier esencialismo, permite comprender otras relaciones con el cuerpo, sus deseos y sentimientos. En cualquier caso, considero que la pregunta por el lugar del cuerpo y su relación con la diferencia sexual sigue siendo una pregunta abierta que posibilitará cada vez más nuevas aproximaciones al feminismo.

\section{Referencias bibliográficas}

Ávila, I. (2014). El nomadismo filosófico de Rosi Braidotti: una alternativa materialista a la metafísica de la presencia. Revista Tabula Rasa, (21), 167-184.

Braidotti, R. (2000). Sujetos nómades. Corporización y diferencia sexual en la teoría feminista contemporánea. Paidós.

Braidotti, R. (2005). Metamorfosis: Hacia una teoría materialista del devenir. Akal. Butler, J. (1999). El género en disputa. El feminismo y la subversión de la identidad. Paidós.

Butler, J. (2002). Cuerpos que importan. Sobre los límites materiales y discursivos del sexo. Paidós.

Cixous, H. (1995). La risa de la medusa. Anthropos.

Hernández, A. (2017). Configuración de la subjetividad nómade. [Tesis de maestría, Universidad del Rosario]. https://repository.urosario.edu.co/ bitstream/handle/10336/13638/HernandezPinzon-AuraMelissa-2017. pdf?sequence $=1 \&$ isAllowed $=\mathrm{y}$

Hernández, A. (2007). Cuerpo a cuerpo con Braidotti y Butler. Riff-raff, (34), 91-99.

Palaisi, M. (2018). El sujeto nómade como contraespacio epistemológico. Enrahonar, an international journal of theoretical and practical reason, (60), 57-73.

Preciado, B. (2012). "Queer": Historia de una palabra. Paroledequeer. http:// paroledequeer.blogspot.com/2012/04/queer-historia-de-una-palabra-por-paul. html 